\title{
Biodiversité des mouches des fruits (Diptera : Tephritidae) en vergers de manguiers de l'ouest du Burkina Faso : structure et comparaison des communautés de différents sites
}

\author{
Sylvain Nafiba OUÉdRAOGo ${ }^{1 *}$, Jean-François VAYSSIÈres ${ }^{2}$, A. Rémy DABIRÉ ${ }^{3}$, Corinne RouLAND-LeFĖVRE ${ }^{4}$
}

\author{
1 Univ. Paris Est, S/C INERA, \\ Farakô Bâ, BP 910, Bobo \\ Dioulasso, Burkina Faso, \\ osylvainn@yahoo.fr \\ 2 CIRAD-Persyst, UPR \\ HortSys, Montpellier 34398, \\ Cedex 5, France / IITA, Biol. \\ Unit Afr., 08 BP 0932 Tri Postal, \\ Cotonou, \\ République du Bénin, \\ j.vayssieres@cgiar.org \\ ${ }^{3}$ Inst. Environ. Rech. Agric. \\ (INERA), Station Farakô Bâ, \\ BP 910, Bobo-Dioulasso, \\ Burkina Faso \\ ${ }^{4}$ IRD-UMR BIOEMCO/IBIOS, \\ 32 Avenue Henri Varagnat,
} F-93143 Bondy Cedex, France

* Correspondance et tirés à part

Reçu le 8 décembre 2010 Accepté le 15 février 2011

Fruits, 2011, vol. 66, p. 393-404 (C) 2011 Cirad/EDP Sciences All rights reserved DOI: $10.1051 /$ fruits/2011054 www.fruits-journal.org

RESUMEN ESPAÑOL, p. 404

\section{Biodiversity of fruit flies (Diptera: Tephritidae) in mango orchards in western Burkina Faso: comparison of structure and communities of different sites.}

Abstract - Introduction. An inventory of Tephritidae species in mango orchards of Western Burkina Faso was conducted from December 2007 to December 2009. Materials and methods. A detection trapping was conducted in mango orchards using the Torula yeast as bait placed in Mac Phail traps. Results and discussion. Identification and enumeration of captured Tephritidae revealed 18 species, nine of the genus Ceratitis, seven of Dacus and two of Bactrocera. Ceratitis cosyra and Bactrocera invadens (respectively 53.02\% and 36.19\% of the whole Tephritids captured) were found to be the dominant species. We have demonstrated a strong similarity between the Tephritidae communities in the different sites investigated (similarity indices varied between $42.86 \%$ and $100 \%$ ); this similarity could be explained by the uniformity of the climatic conditions and by the homogeneity of vegetation in the study area. The proximity of vegetable plots and the diversity of local fruit-tree hosts of Tephritidae around the mango orchards could be the cause of the high species richness we observed. Conclusion. Our study revealed a diversity of Tephritidae in the investigated area which has homogeneous communities of species. These results should be complemented by the identification of species associated with damage to mango and evaluation of their importance. They now suggest the possibility of developing, against these pests, a strategy common to all localities of the investigated area in western Burkina Faso.

Burkina Faso / Mangifera indica / insect control / Tephritidae / Ceratitis cosyra / Bactrocera invadens / pest surveys / traps / biodiversity / population structure

Biodiversité des mouches des fruits (Diptera : Tephritidae) en vergers de manguiers à l'ouest du Burkina Faso: structure et comparaison des communautés de différents sites.

Résumé - Introduction. Un inventaire des espèces de Tephritidae dans des vergers de l'ouest du Burkina Faso a été effectué entre décembre 2007 et décembre 2009. Matériel et méthodes. Un piégeage de détection a été mené dans sept vergers de manguiers en utilisant comme appât de la levure de Torula placée dans des pièges Mac Phail. Résultats et discussion. L'identification et le dénombrement des Tephritidae capturées a permis de recenser 18 espèces : neuf du genre Ceratitis, sept du genre Dacus et deux du genre Bactrocera. Ceratitis cosyra et Bactrocera invadens (respectivement 53,02 \% et 36,19\% de l'ensemble des captures) se sont révélées être les espèces dominantes. Nous avons mis en évidence une forte similarité des communautés de Tephritidae au niveau des différents sites étudiés (coefficients de similitude entre $42,86 \%$ et $100 \%$ ) ; cette similarité pourrait être expliquée d'une part par l'homogénéité des conditions climatiques, d'autre part par celle de la végétation de la zone d'étude. La proximité des parcelles de cultures maraîchères et la diversité des fruitiers locaux hôtes des Tephritidae autour des vergers de manguiers pourraient être à l'origine de la richesse spécifique élevée que nous avons constatée. Conclusion. Notre étude a révélé une diversité de Tephritidae dans la zone d'investigation qui présente des communautés d'espèces homogènes. Ces résultats seront complétés par l'identification des espèces associées aux dégâts sur manguier et à l'évaluation de leur importance. Ils suggèrent dès à présent la possibilité de développer, contre ces ravageurs, une stratégie de lutte commune à toutes les localités de la zone d'étude de l'ouest du Burkina Faso.

Burkina Faso / Mangifera indica / lutte anti-insecte / Tephritidae / Ceratitis cosyra / Bactrocera invadens / enquête organismes nuisibles / piège / biodiversité / structure de la population 


\section{Introduction}

Principale culture fruitière du Burkina Faso (13 500 ha, soit $58 \%$ du verger national), le manguier (Mangifera indica) dont la production annuelle est estimée à plus de $160000 \mathrm{t}$, soit 55,80\% de la production fruitière nationale [1], occupe une place importante dans l'économie rurale du pays. La filière mangue est cependant soumise à diverses contraintes parmi lesquelles les attaques de mouches des fruits (Diptera: Tephritidae). Classées comme ravageurs de quarantaine, les mouches des fruits constituent une contrainte à l'exportation de la mangue, une source de devises pour le pays [2]. Au Burkina Faso, la faible connaissance des Tephritidae du manguier limite le développement d'une stratégie pour le contrôle de leurs dégâts sur la mangue et les autres cultures. En première approche, l'identification des Tephritidae en vergers de manguiers au Burkina Faso devrait contribuer à améliorer la lutte contre ces ravageurs. À la différence de certains pays d'Afrique comme la Côte d'Ivoire [3, 4], le Bénin [5], le Mali [6, 7], la Guinée [8], le Nigéria [9] ou le Togo [10], le Burkina Faso dispose de très peu d'informations sur la biodiversité des Tephritidae infestant localement les manguiers et autres fruitiers cultivés. Les objectifs de notre étude ont donc été (i) de recenser les espèces de Tephritidae présentes dans les vergers de manguiers de l'ouest du Burkina Faso, (ii) de comparer la diversité spécifique des Tephritidae de sept localités de la zone d'étude et (iii) de comparer la diversité des Tephritidae des vergers de l'ouest du Burkina à celle des pays voisins.

\section{Matériel et méthodes}

\subsection{Zone de l'étude}

Notre étude a été effectuée dans sept vergers situés dans les provinces de la Comoé, du Houet et du Kénédougou dans l'ouest du Burkina Faso (tableau I, figure 1), principale zone de production de mangues du pays. Les manguiers y présentent dans le temps un décalage des stades phénologiques avec une fructification précoce à tardive selon les sites (tableau I). Du point de vue climatique, cette zone appartient à la zone soudanienne caractérisée par une saison pluvieuse de 5 à 6 mois. Les hauteurs de précipitations mesurées au cours de l'étude ont varié à Toussiana entre $1301 \mathrm{~mm}$ en 2008 et 1202 mm en 2009 et, à Yéguérésso, entre $1011 \mathrm{~mm}$ en 2008 et $782 \mathrm{~mm}$ en 2009. La température moyenne la plus élevée enregistrée pendant ce suivi a été de $38^{\circ} \mathrm{C}$ notée à Guénako et à Toussiana en 2008. La végétation de cette zone est une savane boisée avec des forêts claires et des îlots de forêts denses sèches et des galeries forestières [11].

\subsection{Inventaire des Tephritidae}

Dans chacun des sept sites considérés, un dispositif de piégeage de détection constitué de quatre pièges Mac Phail [ChemTica Internacional (CTI) S.A., Zeta Industrial Park, La Valencia, Heredia, Costa Rica] a été installé selon la méthode décrite par Vayssières et Sinzogan [12]. La mise en place de ce dispositif est intervenue dans la semaine du 17 au 22 décembre 2007 pour tous les sites sauf pour celui de Guénako où l'installation a eu lieu le 7 janvier 2008. Pour capturer et tuer les adultes mâles et femelles de Tephritidae, la levure de Torula distribuée par CTI a été utilisée comme appât et le dichlorvos [International Parapheromon Shop (IPS), Units 10-15, Meadow Lane, Meadow Lane Industrial Estate, Ellesmere Port, South Wirral, CH65 4TY, England] a été utilisé comme insecticide. Ce dispositif a été suivi entre décembre 2007 et décembre 2009 avec un relevé hebdomadaire des pièges pour récolter des Tephritidae et renouveler la levure de Torula [12]. Pour chaque site, les Tephritidae capturées ont été identifiées et dénombrées.

\subsection{Identification des espèces de Tephritidae}

Les Tephritidae capturées ont été identifiées [13-17] et comparées avec les spécimens d'une collection de référence de mouches 


\section{Tableau I.}

Caractéristiques de sept vergers sites sélectionnés pour étudier la biodiversité des mouches des fruits (Diptera : Tephritidae) en vergers de manguiers de l'ouest du Burkina Faso.

\begin{tabular}{|c|c|c|c|c|c|c|c|}
\hline \multicolumn{2}{|c|}{ Localisation des sites } & \multirow{2}{*}{$\begin{array}{l}\text { Coordonnées } \\
\text { géographiques }\end{array}$} & \multirow{2}{*}{$\begin{array}{l}\text { Altitude } \\
\quad(\mathrm{m})\end{array}$} & \multirow{2}{*}{$\begin{array}{l}\text { Superficie } \\
\text { (ha) }\end{array}$} & \multirow{2}{*}{$\begin{array}{l}\text { Âge des } \\
\text { manguiers } \\
\text { (années) }\end{array}$} & \multirow[t]{2}{*}{ Cultivars présents } & \multirow{2}{*}{$\begin{array}{l}\text { Fructification des } \\
\text { manguiers }\end{array}$} \\
\hline Province & Village & & & & & & \\
\hline \multirow[t]{3}{*}{ Kénédougou } & Koloko & $\begin{array}{l}11^{\circ} 05^{\prime} 51,6^{\prime \prime} \mathrm{N} \\
5^{\circ} 49^{\prime} 31,0^{\prime \prime} \mathrm{E}\end{array}$ & 538 & 6 & 15 & $\begin{array}{l}\text { Amélie, Kent, Keitt, Lippens, } \\
\text { Valencia, Mango vert }\end{array}$ & \multirow{3}{*}{$\begin{array}{c}\text { Tardive : } \\
\text { début des récoltes } \\
\text { en mai }\end{array}$} \\
\hline & Toussian-Bandougou & $\begin{array}{l}10^{\circ} 55^{\prime} 27,7, \mathrm{~N} \\
4^{\circ} 52^{\prime} 19,5, \mathrm{E}\end{array}$ & 486 & 10 & 18 et plus & $\begin{array}{l}\text { Amélie, Brooks, Kent, Keitt, } \\
\text { Lippens, Mango vert }\end{array}$ & \\
\hline & Guénako & $\begin{array}{l}10^{\circ} 56^{\prime} 9,5^{\prime \prime} \mathrm{N} \\
4^{\circ} 49^{\prime} 31,0^{\prime \prime} \mathrm{E}\end{array}$ & 478 & 8 & 16 & $\begin{array}{l}\text { Amélie, Brooks, Kent, } \\
\text { Lippens, Mango vert }\end{array}$ & \\
\hline \multirow[t]{2}{*}{ Comoé } & Soubakaniédougou & $\begin{array}{l}10^{\circ} 28^{\prime} 35,99^{\prime \prime} \mathrm{N} \\
5^{\circ} 00^{\prime} 41,8^{\prime \prime} \mathrm{E}\end{array}$ & 305 & 10 & 18 et 33 & $\begin{array}{l}\text { Amélie, Brooks, Lippens, } \\
\text { Springfels, Mango vert, Sabre }\end{array}$ & \multirow{2}{*}{$\begin{array}{l}\text { Précoce à pleine } \\
\text { saison : } \\
\text { début des récoltes } \\
\text { entre mars et avril }\end{array}$} \\
\hline & Tengrela & $\begin{array}{c}10^{\circ} 39^{\prime} 21,0^{\prime \prime} \mathrm{N} \\
4^{\circ} 51^{\prime} 30,9^{\prime \prime} \mathrm{E}\end{array}$ & 306 & 5 & 16 et 28 & $\begin{array}{l}\text { Amélie, Brooks, Lippens, } \\
\text { Springfels, Mango vert, Sabre }\end{array}$ & \\
\hline \multirow[t]{2}{*}{ Houet } & Toussiana & $\begin{array}{l}10^{\circ} 49^{\prime} 27,4^{\prime \prime} \mathrm{N} \\
4^{\circ} 39^{\prime} 26,1^{\prime \prime} \mathrm{E}\end{array}$ & 480 & 4 & 18 et plus & $\begin{array}{l}\text { Amélie, Brooks, Lippens, } \\
\text { Springfels, Mango vert, Sabre }\end{array}$ & $\begin{array}{l}\text { Précoce: } \\
\text { début des récoltes }\end{array}$ \\
\hline & Yéguérésso & $\begin{array}{c}11^{\circ} 9^{\prime} 29,8^{\prime \prime} \mathrm{N} \\
4^{\circ} 9^{\prime} 53,0 \mathrm{E}\end{array}$ & 352 & 14,7 & 28 et plus & $\begin{array}{c}\text { Amélie, Brooks, Kent, } \\
\text { Lippens, Springfels, Mango } \\
\text { vert, Sabre }\end{array}$ & en mars \\
\hline
\end{tabular}

des fruits du laboratoire de l'Institut international d'agriculture tropicale / Centre de coopération internationale en recherche agronomique pour le développement (IITA / CIRAD) de Cotonou (Bénin).

\subsection{Traitement et analyse des données}

Pour chaque site, la diversité alpha qui mesure la variété et l'abondance des différentes espèces au sein d'une communauté particulière a été calculée. La richesse spécifique des Tephritidae (S) et l'indice de diversité de Shannon-Wiener (H') ont servi à la mesure de cette diversité alpha [18, 19]. Les valeurs de l'indice de diversité de Shannon-Wiener (H') pour les différentes communautés ont été vérifiées à l'aide du test $t$ de Student. L'indice d'équitabilité de Pielou (E) qui tient compte de la répartition des individus entre les espèces [20, 21] a aussi servi à la mesure de la diversité spécifique des différentes communautés.

La diversité bêta qui permet la comparaison de la diversité entre les communautés a été mesurée à l'aide du coefficient de similitude de Jaccard (ISJ) qui tient compte uniquement de la présence des espèces dans la communauté étudiée [22] et de l'indice de similarité de Sorensen ( $\beta$ ) qui est assez proche du premier [23].

\section{Résultats}

\subsection{Diversité alpha}

\subsubsection{Richesse spécifique des Tephritidae}

Dix-huit espèces de Tephritidae appartenant à trois genres Bactrocera spp., Ceratitis spp. et Dacus spp. ont été identifiées dans la zone d'étude. Il s'agit de Bactrocera cucurbitae (Coquillet), Bactrocera invadens Drew et al., Ceratitis anonae Graham, Ceratitis bremii Guérin-Méneville, Ceratitis capitata (Wiedemann), Ceratitis cosyra (Walker), Ceratitis ditissima (Munro), Ceratitis fasciventris (Bezzi), Ceratitis punctata (Wiedemann), Ceratitis quinaria, (Bezzi), Ceratitis silvestrii Bezzi, Dacus bivittatus 


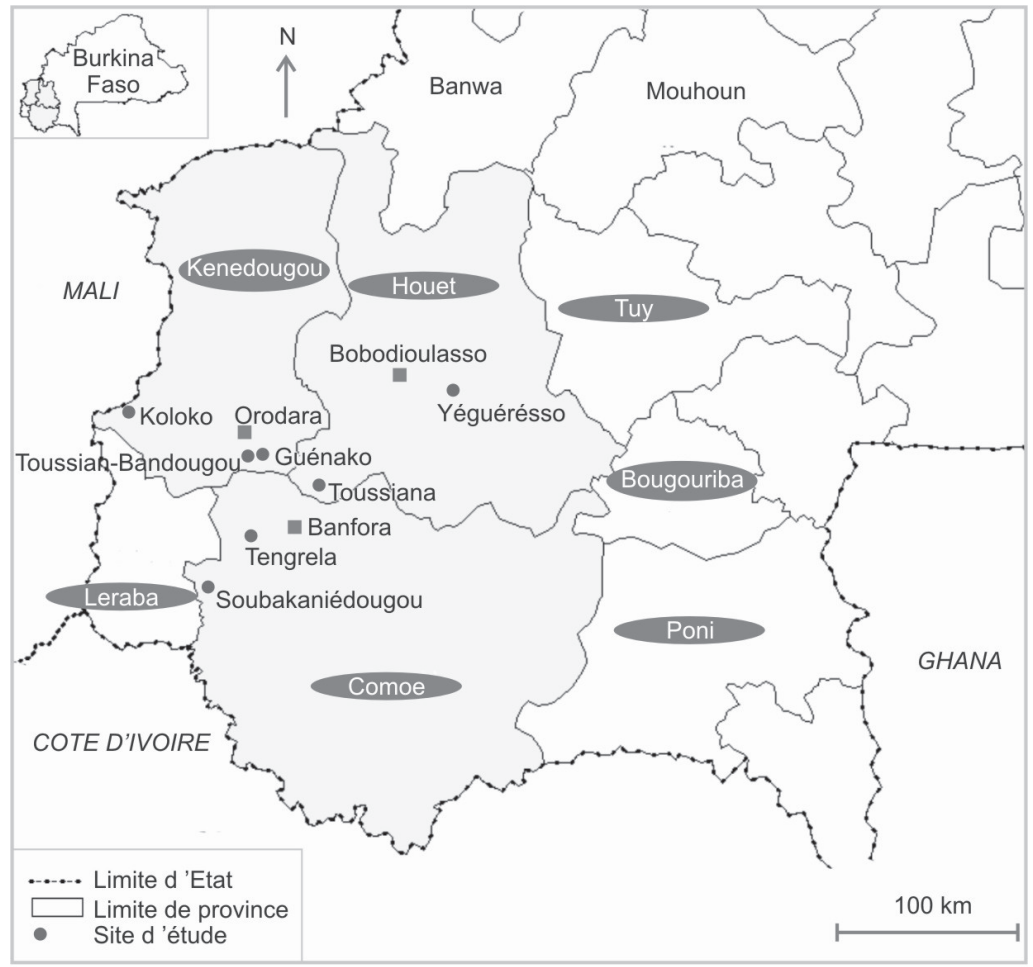

Figure 1.

Localisation des sept sites à l'ouest du Burkina Faso choisis pour étudier la biodiversité des mouches des fruits en vergers de manguiers dans cette zone (source 2010).

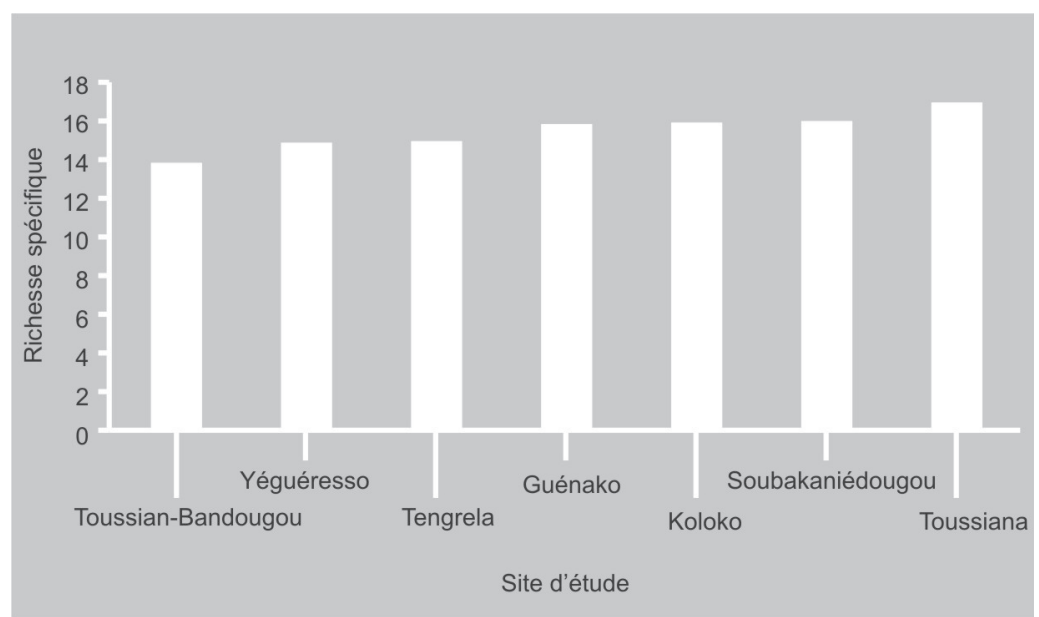

\section{Figure 2.}

Richesse spécifique des Tephritidae capturés entre décembre 2007 et décembre 2009 sur sept sites d'étude localisés en vergers de manguiers à l'ouest du Burkina Faso.
(Bigot), Dacus ciliatus Loew, Dacus langi Curran, Dacus longistylus Wiedemann, Dacus pleuralis Collart, Dacus punctatifrons Karsch et Dacus vertebratus Bezzi. Le genre Ceratitis présente la plus grande richesse spécifique avec neuf espèces identifiées $(50 \%$ de la diversité $\gamma$ ) contre sept pour le genre Dacus (39\% de la diversité $\gamma)$ et deux pour le genre Bactrocera (11\% de la diversité $\gamma$ ). Selon la localité, la distribution des espèces de Tephritidae illustrant la richesse spécifique du site a varié entre 15 pour les localités de Koloko, Tengrela, Toussian-Bandougou et Yéguérésso et 17 pour Toussiana (figure 2). Les sites de Guénako et Soubaka quant à eux ont présenté une richesse spécifique intermédiaire de 16 espèces.

\subsubsection{Diversité spécifique des Tephritidae}

Pour l'ensemble des sites, un total de 117775 adultes de Tephritidae a été capturé dont 51261 mâles (43,52 \% des captures) et 66514 femelles (56,48\% des captures). Les résultats quantitatifs ont révélé la présence de deux espèces dominantes, $C$. cosyra avec 62442 individus $(53,02 \%)$ et B. invadens avec 42628 individus (36,19\%), lesquelles ont représenté environ $89 \%$ des Tephritidae capturées (tableau II). Parmi les seize autres espèces identifiées, seules trois ont présenté une proportion supérieure à $1 \%$ dans l'ensemble des captures ; ce sont $C$. silvestrii $(3,90 \%)$, D. vertebratus $(3,50 \%)$ et C. fasciventris (1,01\%).

L'analyse de la diversité spécifique a montré de faibles valeurs des indices de diversité de Shannon-Wiener ( $\left.\mathrm{H}^{\prime}\right)$ et d'équitabilité de Pielou (E) (tableau III). L'indice de Shannon-Wiener a varié entre $H^{\prime}=0,97$ pour le site de Koloko et $\mathrm{H}^{\prime}=1,57$ pour le site de Yéguérésso ; l'indice de Pielou a varié entre $\mathrm{E}=0,34$ pour Koloko et $\mathrm{E}=0,54$ pour Yéguérésso.

La comparaison des indices de diversité de Shannon-Wiener des différents sites d'étude par le test $t$ de Student a révélé qu'il n'existait pas de différence significative entre les différents sites d'étude. Cependant, l'analyse de la diversité spécifique des mouches des fruits a mis en évidence une contribution inégale des différentes espèces 
Tableau II.

Proportions (en \%) des différentes espèces de Tephritidae capturées au cours d'un inventaire des mouches des fruits présentes sur sept sites occupés par des vergers de manguiers à l'ouest du Burkina Faso, entre décembre 2007 et décembre 2009.

\begin{tabular}{|c|c|c|c|c|c|c|c|c|c|}
\hline Espèce $^{1}$ & Sexe & Guénako & Koloko & Soubakaniédougou & $\begin{array}{l}\text { Toussian- } \\
\text { Bandougou }\end{array}$ & Tengrela & Toussiana & Yéguérésso & $\begin{array}{l}\text { Zone de } \\
\text { l'étude }\end{array}$ \\
\hline \multirow[t]{2}{*}{ B. cucurbitae } & Femelles & 0,031 & 0,066 & 0,526 & 0,15 & 0,293 & 0,076 & 2,399 & 0,272 \\
\hline & Mâles & 0,055 & 0,034 & 0,073 & 0,138 & 0,22 & 0,133 & 1,495 & 0,188 \\
\hline \multirow[t]{2}{*}{ B. invadens } & Femelles & 15,956 & 22,634 & 16,903 & 25,74 & 18,185 & 14,556 & 11,027 & 18,759 \\
\hline & Mâles & 15,511 & 18,342 & 17,839 & 29,27 & 12,642 & 13,971 & 9,081 & 17,435 \\
\hline \multirow[t]{2}{*}{ C. anonae } & Femelles & 0 & 0,003 & 0 & 0 & 0 & 0 & 0 & 0,001 \\
\hline & Mâles & 0 & 0,003 & 0 & 0 & 0 & 0 & 0 & 0,001 \\
\hline \multirow[t]{2}{*}{ C. bremii } & Femelles & 0,039 & 0,074 & 0,073 & 0,282 & 0,311 & 0,045 & 0,088 & 0,104 \\
\hline & Mâles & 0,016 & 0,031 & 0,015 & 0,348 & 0,055 & 0,024 & 0,038 & 0,073 \\
\hline \multirow[t]{2}{*}{ C. capitata } & Femelles & 0,023 & 0,014 & 0,132 & 0,024 & 0,018 & 0,015 & 0 & 0,023 \\
\hline & Mâles & 0,008 & 0,009 & 0,044 & 0 & 0 & 0 & 0 & 0,006 \\
\hline \multirow[t]{2}{*}{ C. cosyra } & Femelles & 38,457 & 32,538 & 26,320 & 23,795 & 25,906 & 37,023 & 22,394 & 31,847 \\
\hline & Mâles & 20,314 & 20,228 & 23,351 & 13,588 & 22,484 & 25,854 & 20,372 & 21,171 \\
\hline \multirow[t]{2}{*}{ C. ditissima } & Femelles & 0,023 & 0,017 & 0,044 & 0,012 & 0 & 0,012 & 0,025 & 0,017 \\
\hline & Mâles & 0,008 & 0,014 & 0 & 0 & 0 & 0,006 & 0 & 0,007 \\
\hline \multirow[t]{2}{*}{ C. fasciventris } & Femelles & 0,594 & 0,616 & 1,067 & 1,267 & 0,695 & 0,327 & 0,276 & 0,632 \\
\hline & Mâles & 0,344 & 0,365 & 0,702 & 0,835 & 0,421 & 0,176 & 0,100 & 0,380 \\
\hline \multirow[t]{2}{*}{ C. punctata } & Femelles & 0,164 & 0,16 & 0,395 & 0,462 & 0,22 & 0,018 & 0,025 & 0,171 \\
\hline & Mâles & 0,148 & 0,148 & 0,219 & 0,288 & 0,22 & 0,006 & 0,025 & 0,127 \\
\hline \multirow[t]{2}{*}{ C. quinaria } & Femelles & 0,234 & 0,245 & 0,117 & 0,054 & 0,055 & 0,270 & 0,025 & 0,193 \\
\hline & Mâles & 0,094 & 0,174 & 0,058 & 0,018 & 0,018 & 0,139 & 0,038 & 0,110 \\
\hline \multirow[t]{2}{*}{ C. silvestrii } & Femelles & 3,843 & 2,140 & 1,360 & 1,861 & 1,518 & 1,948 & 3,680 & 2,262 \\
\hline & Mâles & 2,741 & 1,355 & 1,477 & 0,895 & 1,793 & 1,491 & 3,253 & 1,634 \\
\hline \multirow[t]{2}{*}{ D. bivittatus } & Femelles & 0,008 & 0 & 0 & 0 & 0,018 & 0,003 & 0,389 & 0,029 \\
\hline & Mâles & 0 & 0 & 0 & 0,012 & 0 & 0,003 & 0 & 0,003 \\
\hline \multirow[t]{2}{*}{ D. ciliatus } & Femelles & 0,117 & 0,177 & 0,541 & 0,072 & 1,061 & 0,458 & 1,155 & 0,363 \\
\hline & Mâles & 0,351 & 0,237 & 1,024 & 0,06 & 1,537 & 0,47 & 2,148 & 0,525 \\
\hline \multirow[t]{2}{*}{ D. langii } & Femelles & 0 & 0 & 0,029 & 0 & 0 & 0,003 & 0 & 0,003 \\
\hline & Mâles & 0 & 0 & 0 & 0 & 0,018 & 0 & 0 & 0,001 \\
\hline \multirow[t]{2}{*}{ D. longistylus } & Femelles & 0 & 0 & 0 & 0 & 0 & 0 & 0 & 0 \\
\hline & Mâles & 0,031 & 0 & 0,015 & 0 & 0,055 & 0,003 & 0,013 & 0,008 \\
\hline \multirow[t]{2}{*}{ D. pleuralis } & Femelles & 0,008 & 0,003 & 0,029 & 0,000 & 0,000 & 0,018 & 0,239 & 0,025 \\
\hline & Mâles & 0,008 & 0,000 & 0,015 & 0,000 & 0,037 & 0,000 & 0,239 & 0,020 \\
\hline \multirow[t]{2}{*}{ D. punctatifrons } & Femelles & 0,016 & 0,006 & 0,015 & 0,012 & 0 & 0,052 & 0,327 & 0,042 \\
\hline & Mâles & 0,039 & 0,011 & 0,132 & 0,012 & 0 & 0,055 & 0,578 & 0,071 \\
\hline \multirow[t]{2}{*}{ D. vertebratus } & Femelles & 0,328 & 0,160 & 2,968 & 0,504 & 7,666 & 1,379 & 9,847 & 1,735 \\
\hline & Mâles & 0,492 & 0,194 & 4,518 & 0,300 & 4,555 & 1,466 & 10,726 & 1,764 \\
\hline
\end{tabular}

à la constitution des communautés de Tephritidae dans chaque site avec une forte dominance de $C$. cosyra et $B$. invadens. Ces contributions spécifiques n'ont pas significativement différé selon les sites.

\subsection{Diversité bêta}

Les communautés de Tephritidae des différents sites d'étude ont montré de très grandes similitudes (tableau IV) ; en effet, 


\section{Tableau III. \\ Indices de diversité biologique des Tephritidae capturés sur sept sites d'étude localisés en vergers de manguiers à l'ouest du Burkina Faso.}

\begin{tabular}{lcc} 
Localités & $\begin{array}{c}\text { Indices de Shannon-Wiener } \\
\left(\mathrm{H}^{\prime}\right)\end{array}$ & $\begin{array}{c}\text { Indice d'équitabilité de Piélou }^{2} \\
(\mathrm{E})\end{array}$ \\
\hline Guénako & 1,02 & 0,35 \\
Koloko & 0,97 & 0,34 \\
Soubaka & 1,26 & 0,43 \\
Toussian-Bandougou & 1,02 & 0,35 \\
Tengrela & 1,32 & 0,46 \\
Toussiana & 0,99 & 0,34 \\
Yéguérésso & 1,57 & 0,54
\end{tabular}

${ }^{1}$ L'indice de diversité de Shannon-Wiener est compris entre 0 et $\operatorname{Ln}(S)$ (=2,89 dans cette étude).

${ }^{2}$ L'indice d'équitabilité de Piélou (E) est compris entre 0 et 1.

\section{Tableau IV.}

Indices de diversité bêta des Tephritidae capturés sur sept sites d'étude localisés en vergers de manguiers à l'ouest du Burkina Faso.

a) Indices de Jaccard.

\begin{tabular}{|c|c|c|c|c|c|c|c|}
\hline Site d'étude & Guénako & Koloko & Toussian-Bandougou & Soubakaniédougou & Tengrela & Toussiana & Yéguérésso \\
\hline Guénako & - & 0,94 & 0,67 & 1 & 0,72 & 0,83 & 0,82 \\
\hline Koloko & & - & 0,61 & 0,63 & 0,43 & 0,52 & 0,58 \\
\hline Toussian-Bandougou & & & - & 0,50 & 0,45 & 0,55 & 0,61 \\
\hline Soubakaniédougou & & & & - & 0,63 & 0,83 & 0,63 \\
\hline Tengrela & & & & & - & 0,68 & 0,76 \\
\hline Toussiana & & & & & & - & 0,68 \\
\hline Yéguérésso & & & & & & & - \\
\hline
\end{tabular}

b) Indices de Sorensen.

$\begin{array}{lccccccc}\text { Site d'étude } & \text { Guénako } & \text { Koloko } & \text { Toussian-Bandougou } & \text { Soubakaniédougou } & \text { Tengrela } & \text { Toussiana } & \text { Yéguérésso } \\ \text { Guénako } & - & 0,97 & 0,80 & 1 & 0,84 & 0,91 & 0,90 \\ \text { Koloko } & & - & 0,76 & 0,77 & 0,60 & 0,69 & 0,73 \\ \text { Toussian-Bandougou } & & & - & 0,67 & 0,62 & 0,71 & 0,76 \\ \text { Soubakaniédougou } & & & & - & 0,77 & 0,91 & 0,77 \\ \text { Tengrela } & & & & - & 0,81 & 0,87 \\ \text { Toussiana } & & & & - & -\end{array}$

ces sites hébergent de nombreuses espèces communes. Les sites de Koloko et Tengrela se sont révélés posséder la plus faible similitude avec un indice de Sorensen de 0,60 et un coefficient de similitude (indice de Jacquard) de $43 \%$. En revanche, la plus grande similitude a été notée entre les sites de Guénako et de Soubakaniédougou (indice de
Sorensen $=1$, indice de Jacquard de $100 \%$ ). Sur les 18 espèces recensées, dix ont été couramment rencontrées dans tous les sites et six l'ont été dans au moins cinq des sept sites d'étude. Les espèces les moins fréquemment rencontrées ont été $C$. anonae et D. langii rencontrées respectivement dans un et trois des sept sites d'étude (tableau II). 
Ces résultats traduisent l'homogénéité des communautés de Tephritidae dans les vergers de manguiers de l'ouest du Burkina.

\subsection{Espèces dominantes}

Deux espèces de Tephritidae ont été prédominantes dans les captures des pièges à attractif alimentaire utilisés : $B$. invadens et C. cosyra avec $36,2 \%$ et $53,02 \%$ des captures, respectivement.

\subsubsection{Bactrocera invadens Drew et al.}

Espèce exotique en Afrique, récemment décrite [24], B. invadens est probablement originaire du Sri Lanka (Asie). Cette espèce a été signalée pour la première fois en 2003 en Afrique, au Kenya, d'où elle a connu une très grande expansion aussi bien en Afrique de l'Est [25], qu'en Afrique de l'Ouest [26]. C'est une espèce multivoltine ; les femelles pondent en moyenne 700 oufs et les adultes peuvent vivre environ 3 mois [27]. Par ailleurs, l'espèce est très polyphage ; elle s'attaque à plus d'une trentaine d'espèces fruitières, soit cultivées, soit sauvages, dont le manguier qui en est un hôte primaire [2529].

\subsubsection{Ceratitis cosyra (Walker)}

Encore appelée mango fruit fly, marula fruit fly ou marula fly, C. cosyra est une espèce afrotropicale rencontrée dans toute l'Afrique sub-saharienne jusqu'en Afrique du Sud et à Madagascar [13]. En Afrique de l'Ouest, elle est l'espèce dominante des cératites dans les vergers de manguiers [30]. Espèce multivoltine et polyphage, c'est une espèce qui cause des dégâts importants sur manguier ainsi que sur de nombreuses autres espèces fruitières cultivées ou sauvages [5, 6, 27, 28, 30-33].

\subsection{Autres espèces}

Seize autres espèces de Tephritidae ont été recensées au cours de notre étude (tableau II). Parmi elles, seules trois espèces ont représenté plus de $1 \%$ des captures. Ce sont C. silvestrii Bezzi, C.fasciventris (Bezzi) et D. vertebratus Bezzi.

Couramment rencontrée en Afrique de l'Ouest, C. silvestrii est une espèce afrotro- picale $[13,32]$. Oligophage, elle s'attaque au manguier et à d'autres plantes cultivées et sauvages [27, 29, 30-33].

Espèce afrotropicale, C.fasciventris est présente dans les pays d'Afrique de l'Ouest, du Centre et de l'Est [32]. Différente de C. rosa [34], C.fasciventris est une espèce polyphage qui occasionne des dégâts sur la mangue et sur d'autres espèces fruitières cultivées et sauvages [29-33].

Dacus vertebratus Bezzi, également appelée Jointed pumpkin fly, est une espèce afrotropicale présente en Afrique de l'Ouest, du Centre, et de l'Est, ainsi qu'en Afrique du Sud, à Madagascar et même au Yémen en Asie [13]. Cette mouche des fruits s'attaque à toutes les espèces de cucurbitacées cultivées ou sauvages [35], mais aussi au manguier qui en est un hôte mineur [28]. Sa présence est signalée sur cucurbitacées près des vergers de manguiers au Mali [7].

Treize espèces de Tephritidae ont été rencontrées à des proportions inférieures à $1 \%$ dans les captures effectuées dans les vergers visités ; dans le cadre de notre étude, nous les avons désignées comme espèces rares. Six d'entre elles (C. anonae, C.bremii, C. capitata, C. ditissima, C.punctata et C. quinaria) appartiennent à la tribu des Ceratidini. Les sept autres espèces identifiées (B. cucurbitae, D. bivittatus, D. ciliatus, D. langii, D. longistylus, $D$. pleuralis et D. punctatifrons) sont de la tribu des Dacini. Ceratitis capitata, ou Med fly, est une espèce cosmopolite qui possède une très grande diversité de plantes hôtes [13] et B. cucurbitae, ou Melon fly, bien que faiblement capturée au cours de notre étude, est une espèce d'importance économique couramment rencontrée sur diverses plantes hôtes aussi bien cultivées que sauvages [36].

\section{Discussion}

\subsection{Diversité alpha}

\subsubsection{Richesse spécifique}

L'étude effectuée qui a révélé la présence de 18 espèces de Tephritidae dans les vergers de manguiers de l'ouest du Burkina Faso est 
la première réalisée pour analyser la diversité des mouches des fruits dans ce pays. Parmi ces espèces, quatre (B. invadens, C. cosyra, C. silvestrii et C.quinaria) avaient déjà été signalées sur des mangues infestées. La diversité floristique dans les vergers (plantes adventices) et leur périphérie qui offre une variété de plantes hôtes (ressources) exploitables par les Tephritidae expliquerait cette richesse spécifique élevée [33, 37, 38].

La présence de cucurbitacées sauvages et cultivées dans la flore adventice et dans les jardins maraîchers à proximité des sites étudiés explique la présence, en vergers de manguiers, d'espèces inféodées aux légumes telles que $B$. cucurbitae et $D$. vertebratus [35].

Conduite avec un attractif à faible rayon d'attractivité, notre étude a permis la capture de Tephritidae inféodées au manguier, parmi lesquelles un plus grand nombre d'espèces du genre Ceratitis a été dénombré. Cette situation explique la plus grande richesse spécifique présentée par ce genre par rapport aux autres genres de Tephritidae que nous avons identifiés. Des inventaires réalisés au nord du Bénin et au Mali dans des conditions similaires à notre zone d'étude avaient révélé une aussi grande diversité que celle que nous avons notée dans l'ouest du Burkina Faso [6, 28]. La faible diversité des plantes hôtes de Tephritidae dans les zones d'étude au Nigeria et au Togo pourrait à l'inverse expliquer la faible diversité des Tephritidae notée dans ces pays [9, 10]. La mise en évidence d'une diversité des Tephritidae dans les vergers de manguiers de l'ouest du Burkina Faso suggèrerait de déterminer dans une seconde étape l'importance économique de chacune d'entre elles dans les pertes de mangues.

\subsubsection{Diversité spécifique}

Ceratitis cosyra et Bactrocera invadens se sont révélées être les espèces dominantes de la zone que nous avons étudiée. Le développement des populations d'espèces de Tephritidae dépend des conditions de l'environnement (température et humidité), de la disponibilité des ressources (plantes hôtes) et de leur adéquation avec les besoins des espèces [39, 40].
L'abondance du manguier qui est un des hôtes principaux de C. cosyra dans la zone d'étude et l'adaptation de cette espèce aux conditions environnementales de cette zone $[13,30,31]$ favoriseraient le développement de sa population d'où son abondance dans les captures.

Pour B. invadens, la polyphagie et la capacité de reproduction élevée [26] de l'espèce seraient des facteurs favorables au développement de sa population. Il en est de même de son caractère invasif qui conduirait les populations d'espèces de mouches des fruits natives à se déplacer par le jeu de la compétition interspécifique. Si les espèces dominantes que nous avons identifiées à l'ouest du Burkina Faso semblent semblables à celles observées au nord du Bénin [28], il n'en est pas de même pour celles rapportées par des études réalisées en Côte d'Ivoire, au Nigeria, au Togo et en Tanzanie $[4,9,10,25]$. Ces travaux ont en effet montré que, à l'opposé de B. invadens, C. cosyra n'avait pas le statut d'espèce dominante dans les zones d'étude concernées. Ces différences pourraient s'expliquer par des conditions climatiques différentes et par la variation de la diversité des plantes hôtes de ces zones. En effet, notre étude a été conduite dans une région plus sèche où le manguier est le principal arbre fruitier cultivé favorable au développement de C. cosyra [30].

\subsection{Diversité bêta}

La forte similarité des communautés de Tephritidae des différents sites pourrait être liée à l'homogénéité des conditions climatiques et de la végétation dans la zone que nous avons prospectée à l'ouest du Burkina Faso. En effet, tous les sites de cette étude font partie de la même zone agro-écologique (zone soudanienne) [11]. Annona senegalensis Pers., Bridelia feruginea Benth., Fagara zanthoxyloides (Lam.) Waterman, Parkia biglobosa (Jacq.) R. Br. ex. G. Don, Saba senegalensis (A. Dc.) Pichon, Sarcocephalus latifolius (Smith) Bruce, Sclerocarya birrea (A. Rich) Hochst., Terminalia laxiflora (Engl. \& Diels) et Vitellaria paradoxa Gaertn. F. sont, entre autres, les ligneux que nous avons le plus couramment 
rencontrés aussi bien à l'intérieur des vergers que dans leur voisinage. L'existence d'espèces peu communes dans nos différents sites d'étude, comme C. anonae et $D$. langi, pourrait s'expliquer par l'incapacité de ces espèces à exploiter avantageusement les ressources disponibles. En effet, C. anonae serait un ravageur de la mangue plus important dans les zones soudaniennes plus humides que dans les zones sahéliennes [6]. L'homogénéité des communautés de Tephritidae dans les vergers de manguiers de l'ouest du Burkina Faso soutiendrait la possibilité de développer, pour toute la zone, une stratégie unique de lutte contre ces ravageurs du manguier.

\section{Conclusion}

L'étude que nous avons menée pour identifier la biodiversité des mouches des fruits présentes en vergers de manguiers dans l'ouest du Burkina Faso a mis en évidence la présence d'une grande diversité de Tephritidae. Dix-huit espèces relevant des genres Bactrocera (deux espèces), Ceratitis (neuf espèces) et Dacus (sept espèces) ont été identifiées. Ces différentes espèces contribuent inégalement à la constitution des communautés de Tephritidae, comme le montrent les faibles valeurs des indices de diversité de Shannon-Wiener et d'équitabilité de Pielou.

Mieux adaptée aux conditions de notre zone d'étude, C. cosyra est l'une des deux espèces dominantes que nous avons identifiées sur manguier dont c'est l'un de ses principaux hôte. L'autre espèce dominante est une espèce invasive en Afrique, B. invadens capturée en mai 2005 à Bobo Dioulasso pour la $1^{\text {re }}$ fois $[26,41]$. Les communautés de Tephritidae se sont révélées homogènes avec des coefficients de similitude variant entre $42,86 \%$ et $100 \%$.

Cet inventaire, premier du genre effectué au Burkina Faso, nous permet de connaître la composition spécifique des communautés de Tephritidae dans la zone d'investigation et de déterminer l'abondance de chacune des espèces.
L'homogénéité de ces communautés de Tephritidae mise en évidence permettrait d'envisager le développement d'une stratégie de lutte commune dans la zone de l'étude. Un travail est en cours qui fait suite à ces premiers résultats; il porte sur un inventaire des espèces de Tephritidae à partir de l'étude de mangues infestées et il devrait permettre d'évaluer les dégâts occasionnés sur ces fruits en fonction du cultivar utilisé et de la période d'échantillonnage. Dans ce contexte, plus de 19760 mangues ont été collectées, puis mises en incubation. Les résultats obtenus feront l'objet d'un prochain article.

\section{Remerciements}

Au terme de cette étude, nous remercions les producteurs de mangues de la zone ouest du Burkina pour leur disponibilité et l'équipe "terrain mouches des fruits " de l'INERA, Farakô Ba, Burkina Faso, pour son appui à la collecte des données. De même, nous remercions tous ceux qui ont lu et corrigé ce manuscrit. Cette étude a été réalisée avec l'appui financier de la fondation italienne Un Raggio Di Luce et le soutien de la Coopération française au Burkina à travers l'ambassade de France à Ouagadougou que nous remercions particulièrement.

\section{Références}

[1] Diallo Y., La lutte du PAFASP contre la mouche des fruits, in: La lutte régionale contre les mouches des fruits en Afrique subsaharienne, Coleacp / Cirad, Lett. Inf. no 5, 2010, Paris, France, http:// www.coleacp.org/fr/system/files/file/ COLEACP/LE_2010_05_FR.pdf.

[2] Guichard C., Interceptions de mangues d'Afrique à l'entrée de l'UE pour cause de mouches des fruits (Tephritidae), in : La lutte régionale contre les mouches des fruits en Afrique subsaharienne, Coleacp / Cirad, Lett. Inf. no 1, 2009, Paris, France, http:// www.coleacp.org/fr/system/files/file/ coleacp/lutte_regionale_contre_les_ mouches_des_fruits_et_legumes_nr1.pdf. 
[3] Hala N., Quilici S., Gnago A.J., N'Depo O.R., N'Da Adopo A., Kouassi P., Allou K., Status of fruit flies (Diptera: Tephritidae) in Côte d'Ivoire and implications for mango exports, in: Fruit flies of economic importance: from basic to applied knowledge, Proc. 7th Int. Symp. Fruit Flies of Economic Importance, Salvador, Brazil, 10-15 September 2006, pp. 233-239.

[4] Ossey R.N., Hala N., Allou K., Aboua L.R., Kouassi K.P., Vayssières J.-F., De Meyer M., Abondance des mouches des fruits dans les zones de production fruitières de Côte d'Ivoire : dynamique des populations de Bactrocera invadens (Diptera : Tephritidae), Fruits 64 (2009) 313-324.

[5] Vayssières J.-F., Georgen G., Lokossou O., Dossa P., Akponon C., A new Bactrocera species in Benin among mango fruit fly (Diptera: Tephritidae) species, Fruits 60 (2005) $1-7$.

[6] Noussourou M., Diarra B., Mouches des fruits au Mali : Bioécologie et possibilités de lutte intégrée, Sahel IPM 6 (1995) 2-13.

[7] Vayssières J.-F., Sanogo F., Noussourou M., Inventaire des espèces de mouches de fruits (Diptera: Tephritidae) inféodées au manguier au Mali et essai de lutte raisonnée, Fruits 59 (2004) 1-14.

[8] Vayssières J.-F., Kalabane S., Inventory and fluctuations of the catches of Diptera Tephritidae associated with mangoes in costal Guinea, Fruits 55 (2000) 259-270.

[9] Umeh V.C., Garcia L.E., De Meyer M., Fruit flies of citrus in Nigeria: species diversity, relative abundance and spread in major producing areas, Fruits 63 (2008) 145-153.

[10] Amévoin K., Sanbena B.B., Nuto Y., Gomina M., De Meyer M., Glitho I.A., Les mouches des fruits (Diptera : Tephritidae) au Togo: inventaire, prévalence et dynamique des populations dans la zone urbaine de Lomé, Int. J. Biol. Chem. Sci. 3 (5) (2009) 912-920.

[11] Anon., Atlas de l'Afrique: Burkina Faso, Lerebourgs Pigeonnière A., Ménager M.T., Meunier-Nikiema A. (Eds.), Les atlas de I'Afrique, Ed. Jaguar, Paris, France, 2005.

[12] Vayssières J.-F., Sinzogan A., Piégeage de détection des mouches des fruits, IITACIRAD, Fiche Tech. WAFFI no 3, Cotonou, Benin, 2008, 4 p.

[13] Carroll L.E., White I.M., Freidberg A., Norrbom A.L., Dallwitz M.J., Thompson F.C.,
Pest fruit flies of the world: identification, descriptions, illustrations, and information retrieval, USDA-ARS, USA, 2002, http:// delta-intkey.com/

[14] White I.M., Taxonomy of the Dacinae (Diptera: Tephritidae) of Africa and the Middle East, Entomol. Soc. South. Afr., Afr. Entomol., Mem. no 2, Hatfield, S. Afr., 2006.

[15] White I.M., Elson-Harris M., Fruit flies of significance: their identification and bionomics, CAB Int., Lond., U.K. \& ACIAR, Canberra, Austr., 1992.

[16] De Meyer M., Revision of the subgenus Ceratitis (Pardalaspis) Bezzi, 1918 (Diptera, Tephritidae, Ceratitini), Syst. Entomol. 21 (1996) 15-26.

[17] De Meyer M., Revision of the subgenus Ceratitis (Ceratalaspis) Hancock (Diptera: Tephritidae), Bull. Entomol. Res. (88) 3 (1998) 257-290.

[18] Jayaraman K., Manuel de statistique pour la recherche forestière, Kerala For. Res. Inst. Peechi, Thrissur, Kerala, Inde, 2000.

[19] Peet R.K., The measurement of species diversity, Annu. Rev. Ecol. Syst. 5 (1974) 285-307.

[20] Frontier S., Pichod-Viale D., Écosystèmes : structure, fonctionnement, évolution, Masson Fr., Collect. Ecol. 21, 1991.

[21] Brugneaux S., Pierret L., Bouchon C., Bouchon-Navarro Y., Portillo P., Louis M., Suivi de l'état de santé des récifs coralliens de la Martinique, campagne 2001-2003, Obs. Milieux Marins Martin., Univ. Antill. Guyane, Fort de France, France, 2004.

[22] Kiéma S., Élevage extensif et conservation de la diversité biologique dans les aires protégées de l'Ouest Burkinabé. Arrêt sur leur histoire, épreuves de la gestion actuelle, état et dynamique de la végétation, Univ. Orléans, Thèse, Orléans, France, 2007.

[23] Sørensen T.A., A method of establishing groups of equal amplitude in plant sociology based on similarity of species content, and its application to analyses of the vegetation on Danish commons, K. Dan. Vidensk. Selsk. Biol. Skr. 5 (1948) 1-34.

[24] Drew R.A.I., Tsuruta K., White I.M., A new species of pest fruit fly (Diptera: Tephritidae: Dacinae) from Sri Lanka and Africa, Afr. Entomol. 13 (2005) 149-154. 
[25] Mwatawala M.W., De Meyer M., Makundi R.H., Maerere A.P., Biodiversity of fruit flies (Diptera, Tephritidae) in orchards in different agro-ecological zones of the Morogoro region, Tanzania, Fruits 61 (2006) 321-332.

[26] Vayssières J.-F., Sinzogan A., BokononGanta A., La nouvelle espèce invasive de mouches des fruits: Bactrocera invadens Drew Tsuruta \& White, IITA-CIRAD, Fiche Tech. WAFFI no 2, Cotonou, Benin, 2008, $4 \mathrm{p}$.

[27] Ekesi S., Nderitu P.W., Rwomushana I., Field infestation, life history and demographic parameters of Bactrocera invadens Drew Tsuruta \& White, a new invasive fruit fly species in Africa, Bull. Entomol. Res. 96 (2006) 279-386.

[28] Vayssières J.-F., Korie S., Ayegnon D., Correlation of fruit fly (Diptera Tephritidae) infestation of major mango cultivars in Borgou (Benin) with abiotic and biotic factors and assessment of damage, Crop Prot. 28 (2009) 477-488.

[29] Mwatawala M.W., De Meyer M., Makundi R.H., Maerere A.P., Seasonality and host utilisation of the invasive fruit fly, Bactrocera invadens (Diptera, Tephritidae), in central Tanzania, J. Appl. Entomol. 130 (2006) 530537.

[30] Vayssières J.-F., Sinzogan A., BokononGanta A., Les mouches des fruits du genre Ceratitis (Diptera: Tephritidae) en Afrique de I'Ouest, IITA-CIRAD, Fiche Tech. WAFFI no 1, Cotonou, Benin, 2008, 4 p.

[31] Ouédraogo S.N., Étude des attaques de la mangue (Mangifera indica) par les mouches des fruits (Diptera : Tephritidae) dans la province du Kénédougou (ouest du Burkina Faso), Univ. Polytech. Bobo-Dioulasso, Mém. DEA Entomol., Bobo-Dioulasso, Burkina Faso, 2007, 57 p.

[32] Vayssières J.-F., Sinzogan A., Adandonon A., Gamme de plantes-hôtes cultivées et sauvages pour les principales espèces de mouches des fruits au Bénin, IITA-CIRAD,
Fiche Tech. WAFFI no 8, Cotonou, Benin, 2009, 4 p.

[33] Ouédraogo S.N., Vayssières J.-F., Dabiré R.A., Rouland-Lefèvre C., Fruitiers locaux hôtes des mouches des fruits (Diptera: Tephritidae) inféodées au manguier dans l'ouest du Burkina Faso : identification et taux d'infestation, Fruit Veg. Cereal Sci. Biotechnol. 4 (Spec. Issue 1) (2010) 36-41.

[34] De Meyer M., On the identity of the natal fruit fly Ceratitis rosa Karsch (Diptera, Tephritidae), Bull. Inst. R. Sci. Natl. Belg. Entomol. 71 (2001) 55-62.

[35] Bordat D., Arvanitakis L., Arthropodes des cultures légumières d'Afrique de l'Ouest, Centrale, Mayotte et Réunion, Ed. Cirad, Montpellier, France, 2004.

[36] Vayssières J.-F., Rey J.-Y., Traoré L., Distribution and host plants of Bactrocera cucurbitae in West and Central Africa, Fruits 62 (2007) 391-396.

[37] Clarke A.R, Armstrong K.F., Carmichael A.E., Milne J.R., Raghu S., Roderick G.K., Yeates D.K., Invasive phytophagus pests arising through a recent tropical evolutionary radiation: The Bactrocera dorsalis complex of fruit flies, Annu. Rev. Entomol. 50 (2005) 293319.

[38] Metcalf R.L., Chemical ecology of Dacinae fruit flies (Diptera: Tephritidae), Ann. Entomol. Soc. Am. 83 (1990) 1017-1030.

[39] Bateman M.A., The ecology of fruit flies, Annu. Rev. Entomol. 17 (1972) 493-518.

[40] Raghu S., The autecologie of Bactrocera cacuminata (Hering) (Diptera: Tephritidae: Dacinae): Functional significance of resources, Griffith Univ., Thesis, Griffith, Aust., 2002, 241 p.

[41] Vayssières J.-F., Sinzogan A., Adandonon A., New developments on the fruit fly issue in West Africa, in: Fighting fruit flies regionally in sub-Saharan Africa, Coleacp-Cirad, Inf. Letter 11 (2010) 3. 
Biodiversidad de las moscas de la fruta (Diptera : Tephritidae) en huertos de mangos, al oeste de Burkina Faso : estructura y comparación de las comunidades de los diferentes terrenos.

Resumen - Introducción. Se realizó un inventario de las especies de Tephritidae en huertos al oeste de Burkina Faso, entre diciembre de 2007 y diciembre de 2009. Material y métodos. Se efectuó una instalación de trampas de detección, en siete huertos de mangos, empleando como cebo la levadura de tórula, que se colocó en trampas Mac Phail. Resultados y discusión. La identificación y el conteo de las Tephritidae capturadas permitió inventariar 18 especies: nueve del género Ceratitis, siete del género Dacus y dos del género Bactrocera. Ceratitis cosyra y Bactrocera invadens (respectivamente el 53,02\% y el 36,19 \% del conjunto de las capturas) resultaron ser las especies dominantes. Destacamos una fuerte similitud de las comunidades de Tephritidae a nivel de los diferentes terrenos estudiados (coeficientes de similitud entre el $42,86 \%$ y el $100 \%$ ); esta similitud podría explicarse, por un lado, por la homogeneidad de las condiciones climáticas, por otro lado, por la de la vegetación de la zona de estudio. La proximidad de las parcelas de la horticultura comercial y la diversidad de los árboles frutales locales, huéspedes de los Tephritidae, alrededor de los huertos de mangos, podrían ser el origen de la elevada riqueza específica que constatamos. Conclusión. Nuestro trabajo mostró una diversidad de Tephritidae en la zona de investigación que presenta comunidades de especies homogéneas. Dichos resultados se completarán mediante la identificación de las especies asociadas tanto a los estragos en el mango, como a la evaluación de su importancia. Además, sugieren, de ahora en adelante, la posibilidad de desarrollar contra esta plaga una estrategia de lucha común en todas las localidades de la zona de estudio del oeste de Burkina Faso.

Burkina Faso / Mangifera indica / control de insectos / Tephritidae / Ceratitis cosyra / Bactrocera invadens / encuestas sobre plagas / trampas / biodiversidad / estructura de la población 